\title{
Montreal Cognitive Assessment (MoCA): Validation Study for Vascular Dementia
}

\author{
Sandra Freitas, ${ }^{1,2}$ Mário R. Simões, ${ }^{1,2}$ Lara Alves, ${ }^{1}$ Margarida Vicente, ${ }^{1}$ AND Isabel Santana ${ }^{3}$ \\ ${ }^{1}$ Faculty of Psychology and Educational Sciences, University of Coimbra, Coimbra, Portugal \\ ${ }^{2}$ Centro de Investigação do Núcleo de Estudos e Intervenção Cognitivo Comportamental (CINEICC), Coimbra, Portugal \\ ${ }^{3}$ Neurology Department of the Coimbra University Hospital \& Faculty of Medicine, University of Coimbra, Coimbra, Portugal
}

(Received November 16, 2011; Final Revision May 8, 2012; Accepted May 8, 2012)

\begin{abstract}
The Montreal Cognitive Assessment (MoCA) is a brief instrument developed for the screening of milder forms of cognitive impairment, having surpassed the well-known limitations of the MMSE. The aim of the present study was to validate the MoCA as well as its short version, which was proposed by the NINDS-CSN VCI Harmonization Standards for screening Vascular Dementia (VaD) patients. The results, based on a homogeneous sample of $34 \mathrm{VaD}$ patients, indicate that the MoCA is a psychometrically valid and reliable instrument for cognitive screening in $\mathrm{VaD}$ patients, showing excellent discriminant validity. Both the full and short versions of the MoCA had excellent diagnostic accuracy in discriminating $\mathrm{VaD}$ patients, exhibiting an area under curve (AUC) higher than the MMSE [AUC(MoCA full version $)=.950 ; 95 \% \mathrm{IC}=.868-.988 ;$ AUC $($ MoCA short version $)=.936 ; 95 \% \mathrm{IC}=.849-.981 ;$ AUC $(\mathrm{MMSE})=.860$; $95 \%$ IC $=.754-.932]$. With a cutoff below 17 on the MoCA full version and 8 on the short version, the results for sensitivity, specificity, positive and negative predictive values, and classification accuracy were superior compared to the MMSE. In conclusion, both versions of the MoCA are valid, reliable, sensitive and accurate screening instruments for $\mathrm{VaD}$ patients. (JINS, 2012, 18, 1031-1040)
\end{abstract}

Keywords: Neuropsychological test, Validation studies, Geriatric assessment, Cognition, Vascular Dementia, Alzheimer disease

\section{INTRODUCTION}

In recent years, the nosologic concept of vascular cognitive impairment and dementia has generated a huge controversy and disagreement regarding diagnostic criteria. The existence of many cases of cognitive deficit resulting from cerebrovascular disease but not fulfilling the criteria for dementia has led to the recent emergence of a broader concept named Vascular Cognitive Impairment (VCI; Hachinski \& Bowler 1993; O’Brien, Reisberg, \& Erkinjuntti, 2003). In this context, three diagnostic categories are mainly proposed: Vascular Mild Cognitive Impairment (vMCI, also known as Vascular Cognitive Impairment No Dementia, VCIND), Vascular Dementia (VaD), and mixed dementia (Moorhouse \& Rockwood, 2008; O'Brien et al., 2003).

The concept of $\mathrm{VaD}$, which is classified as a VCI subtype, was introduced in the 1990s (Román et al., 1993), replacing

Correspondence and reprint requests to: Sandra Freitas, Faculty of Psychology and Educational Sciences, University of Coimbra, Rua do Colégio Novo, Apartado 6153, 3001-802 Coimbra, Portugal. E-mail: sandrafreitas0209@gmail.com the previous concepts of Cerebral Arteriosclerosis and Multi-infarct dementia (Hachinski, Lassen, \& Marshall, 1974). This concept's evolution was associated with the proposal of new diagnostic criteria for Vascular Dementia by the National Institute of Neurological Disorders and Stroke - Association Internationale pour la Recherche et l'Enseignement en Neurosciences (NINDS-AIREN; Román et al., 1993). These criteria were organized and further operationalized by van Straaten and collaborators (2003), considering three fundamental axes of diagnosis: (a) presence of dementia, confirmed by neuropsychological assessment; (b) clinical and imaging evidence of cerebrovascular disease; and (c) establishment of a causal relationship between the vascular lesions and the emergence or aggravation of the cognitive deficits. The harmonization and coverage of clinical, neuroimaging, and anatomic-pathologic data makes these diagnostic criteria much more objective and selective than previous ones, namely the old Hachinski ischemic score (Hachinski et al., 1975), explaining why they have gathered more consensus in clinical practice, becoming the most widely used in research (Santana, 2006). 
Epidemiological studies, although not consistent, have generally reported that $\mathrm{VaD}$ is the second most common cause of dementia, after Alzheimer's disease (AD) (Canadian Study of Health and Aging Working Group, 1994; Lobo, Martinez-Lage et al., 2000). In Europe, the prevalence rate is approximately $16 / 1000$ in people over 65 years old, representing approximately $20 \%$ of all dementia cases (Ikeda et al., 2001; Lobo, Launer et al., 2000). Moreover, in some Asian countries, $\mathrm{VaD}$ appears to be more prevalent than $\mathrm{AD}$ (Ikeda et al., 2001; Ueda, Kawano, Hasuo, \& Fujishima, 1992). Both prevalence and incidence of $\mathrm{VaD}$ and $\mathrm{AD}$ increase exponentially with age, and even though the increase in $\mathrm{VaD}$ seems to be less steep than for $\mathrm{AD}$, the prevalence rate for individuals over 100 years old surpasses 52/1000 (Jorm \& O'Brien, 2004; Lobo, Launer et al., 2000).

This high prevalence of $\mathrm{VaD}$, highlight the importance of an accurate clinical identification of this disease. Moreover, this purpose is generally acknowledged to be difficult, largely because of the clinical features of VaD (Garrett, Paul, Libon \& Cohen, 2004; Reed et al., 2004). The clinical features of the vascular cognitive impairment reflect the location, number, and extent of underlying ischemic, hemorrhagic, or hypoperfusion lesions (Desmond, 2004; Ueda et al., 1992). As a result of this heterogeneity in mechanisms, vascular territory involvement and location, cognitive impairment in $\mathrm{VaD}$ is highly variable and potentially affects all cognitive domains. Despite this, the preponderant neuropsychological features of $\mathrm{VaD}$ patients point to an overall profile of cognitive dysfunction that is frontally located in the brain, due to the early involvement of executive cortico-subcortical circuits (O'Brien et al., 2003; Román, 2003; Román \& Royall, 1999). The dysexecutive syndrome has been recurrently associated with the vascular clinical conditions (Desmond, 2004; Lamar, Price, Giovannetti, Swenson, \& Libon, 2010). Additionally, cortical functions such as language, calculation, and orientation tend to be relatively preserved, in contrast with AD patients (Looi \& Sachdev, 1999; Román \& Royall, 1999). Deficits in recent episodic memory are usually less severe in $\mathrm{VaD}$ patients than in degenerative dementias, while concentration difficulties, working memory dysfunction, and psychomotor slowing are early and prominent symptoms in $\mathrm{VaD}$ (Looi \& Sachdev, 1999; O’Brien et al., 2003; Román, 2003).

An additional factor for this difficult to diagnose $\mathrm{VaD}$ refers to the insufficient breadth of representation of the cognitive abilities relevant to cognitive impairment associated with the disease in cognitive assessment tools, an essential condition for the accurate cognitive recognition and assessment (Mungas, Reed, \& Kramer, 2003). In fact, traditional screening tools frequently do not include measures of important domains initially impaired in $\mathrm{VaD}$, mainly the executive functions, or the evaluation is very superficial using low complex tasks. This is the case of the Mini Mental State Examination (MMSE; Folstein, Folstein, \& McHugh, 1975), which is the most widely used cognitive screening instrument for dementia, despite its well-known limitations; namely, it has low sensitivity to milder forms of cognitive impairment and does not evaluate executive functioning
(Freitas, Santana, \& Simões, 2010; Ihl, Frölich, Martin, \& Maurer, 1992; Tombaugh \& McIntyre, 1992; Wind et al., 1997). These issues, according to several experts, are especially relevant for dementias such as $\mathrm{VaD}$ that present prominent executive impairment (Hachinski et al., 2006; Martinić-Popović, Šerić, \& Demarin, 2006; Pendlebury, Cuthbertson, Welch, Mehta, \& Rothwell, 2010; Pirscoveanu et al., 2009; Román, 2003).

The Montreal Cognitive Assessment (MoCA; Nasreddine et al., 2005) is a cognitive screening test specifically developed for the screening of milder forms of cognitive impairment. Surpassing the limitations of the MMSE and proving be a more useful, sensitive and accurate measure, the MoCA is now recognized as one of the best cognitive screening tests (Gauthier et al., 2011; Ismail, Rajji, \& Shulman, 2009; Jacova, Kertesz, Blair, Fisk, \& Feldman, 2007; Lonie, Tierney, \& Ebmeier, 2009). Currently, the MoCA is an extensively validated screening tool for many disorders; it displays good overall psychometric properties and improved sensitivity to cognitive decline, which contributed to its rapid international recognition. This improved sensitivity in relation to other measures is related to the MoCA's more comprehensive assessment of major cognitive domains, including executive function, short-term memory, languages abilities, and visuospatial processing. Considering the qualities and adequacy of the MoCA, the neuropsychology working group of the NINDS-CSN VCI Harmonization Standards criteria (Hachinski et al., 2006), recommends using the abbreviated version of the MoCA (12 items-hereinafter referred as MoCA short version) for brief cognitive screening in vascular patients in special settings such as primary health care, large epidemiological studies or clinical trials. This proposed 5-min protocol may be administered by telephone and is composed of three subtests: a five-word immediate and delayed recall test, a six-item orientation task, and a phonemic fluency test.

Several studies have been conducted to investigate cerebrovascular and cardiovascular diseases using the MoCA full version (Aggarwal \& Kean, 2010; Cumming, Bernhardt, \& Linden, 2011; Dong et al., 2010; Ihara, Okamoto, \& Takahashi, 2012; Pendlebury et al., 2010; Pirscoveanu et al., 2009; Wong et al., 2008; 2009). All of these studies indicate that the MoCA is a psychometrically valid, reliable and sensitive cognitive screening test for the mentioned clinical conditions. Thus far, however, there are few previous studies using a homogeneous group of $\mathrm{VaD}$ patients to confirm those results and no studies of validation of the MoCA short version.

The aim of the present study was to investigate the validity of the MoCA (Nasreddine et al., 2005; Simões et al., 2008) for cognitive screening of $\mathrm{VaD}$ patients. The psychometric properties and diagnostic accuracy of the MoCA was analyzed and the optimal cutoff points to detect $\mathrm{VaD}$ patients were established. In parallel, the same analysis was conducted for the short version of the MoCA proposed by NINDS-CSN VCI Harmonization Standards (Hachinski et al., 2006). Furthermore, we also aimed to investigate the cognitive performance of the $\mathrm{VaD}$ patients on the MoCA's cognitive domains and analyze the differences with comparison to $\mathrm{AD}$ patients. 


\section{METHODS}

\section{Participants and Procedures}

The study sample was composed of 102 participants distributed between three subgroups: (I) a $\mathrm{VaD}$ group with 34 patients, (II) an $\mathrm{AD}$ group with 34 patients, and (III) a Control group with 34 cognitively healthy adults. The demographic data of the participants in each group are summarized in Table 1.

The $\mathrm{VaD}$ patients were recruited between January 2009 and June 2011 at the Dementia Clinic of the Neurology Department of Coimbra University Hospital (Coimbra, Portugal) and included only patients with a diagnosis of probable $\mathrm{VaD}$, based on international criteria (Román et al., 1993). Patients with Vascular Mild Cognitive Impairment (vMCI), also called Vascular Cognitive Impairment no Dementia (VCIND), or with mixed dementia were not included. Eligibility was based on a comprehensive clinical evaluation, which included a neuropsychological and imaging study, and the final diagnosis was established by a multidisciplinary team consensus composed by two neurologists, two neuropsychologists and two neuroradiologists with expertise in dementia investigation.

The AD group included the patients with a diagnosis of probable $\mathrm{AD}$, as previously established by a multidisciplinary team consensus using international criteria (American Psychiatric Association, 2000; McKhann et al., 1984). This diagnosis subgroup excluded patients with significant vascular disease, using either clinical or imaging evaluations. This group was selected from among the AD patients of the Dementia Clinic of the Neurology Department of Coimbra University Hospital (Coimbra, Portugal) to match $\mathrm{VaD}$ patients on gender, age, educational level, and severity of cognitive decline, as assessed by the MMSE (Folstein et al., 1975).

All patients were clinically examined by a neurologist (I.S.), and a standard investigation was always performed, including routine laboratory exams and analyses (Apolipoprotein $\mathrm{E}$ [APOE] genotyping and imaging studies: structural (computed tomography [CT] and/or magnetic resonance imaging [MRI]) and functional (single photon emission CT [SPECT]). PET and cerebrospinal fluid analyses were carried

Table 1. Demographic and clinical characteristics of the subgroups

\begin{tabular}{lccc}
\hline \hline & \multicolumn{1}{c}{$\mathrm{VaD}$} & $\mathrm{AD}$ & Control \\
\hline$n$ & 34 & 34 & 34 \\
Gender & $12(35.3)$ & $12(35.3)$ & $12(35.3)$ \\
Age & $73.21 \pm 7.85$ & $74.00 \pm 7.36$ & $73.65 \pm 7.40$ \\
Educational Level & $4.97 \pm 2.747$ & $5.09 \pm 2.54$ & $5.09 \pm 2.82$ \\
MMSE score & $24.06 \pm 4.01$ & $23.24 \pm 4.21$ & $27.94 \pm 1.41$ \\
MoCA score & $13.06 \pm 4.62$ & $11.47 \pm 4.24$ & $22.97 \pm 3.38$ \\
\hline \hline
\end{tabular}

Note. Gender is characterized by female's $n$ and respective percentage (\%). Data of other variables are presented as mean \pm standard deviation. $\mathrm{VaD}=$ group of patients with Vascular Dementia; $\mathrm{AD}=$ group of patients with Alzheimer's disease; Control = group of cognitively healthy adults; MoCA $=$ Montreal Cognitive Assessment (maximum score $=30$ ); MMSE $=$ Mini Mental State Examination (maximum score $=30)$. out more restrictively, although they were considered in younger patients and whenever the suspicion of an inflammatory, infectious, or neoplastic brain involvement was raised. Structural imaging studies, namely CT or MRI, were fundamental in the differential diagnosis of dementia patients in this study. In accordance with the inclusion criteria for $\mathrm{VaD}$ and the exclusion criteria for $\mathrm{AD}$, the following findings were relevant: (1) evidence of large cortico-subcortical infarcts; (2) extensive periventricular (PVL) or subcortical white matter lesions (SCWML), affecting more than $25 \%$ of the volume of the supra-tentorial white matter (WML), based on a CT or MRI volumetric qualitative analysis; (3) uni- or bilateral thalamic strokes; (4) lacune in the head of the caudate nucleus or in the inferior genu of the internal capsule; (5) more than two lacunes. At the outset of this study, the following were also considered exclusion criteria for patients' selection: (1) an unstable clinical condition, with significant comorbidities; (2) high dementia severity (only including patients with CDR $\leq 2$ and MMSE $\geq 12$ points); (3) recent pharmacotherapy changes (patients on stable medication ( $>6$ months) with cholinesterase inhibitors or psychotropic drugs were allowed to enter the study, but recent dose changes of these drugs, as well as introduction of medication with anti-cholinergic effect were considered an exclusion criteria); (4) recent psychiatric comorbidity (clinically diagnosed in the 6 months before the current neuropsychological evaluation); and (5) significant motor, visual or auditory deficits, all of which may influence neuropsychological assessment. For each patient considered suitable for the study at the time of data collection, a diagnosis was recorded in the clinical file by the neurologist.

All patients also underwent a comprehensive neuropsychological assessment battery including the following instruments: the MMSE (Folstein et al., 1975), the Alzheimer's Disease Assessment Scale (ADAS; Rosen, Mohs, \& Davis, 1984), the Clinical Dementia Rating scale (CDR; Hughes, Berg, Danziger, Coben, \& Martin, 1982), the Irregular Word Reading Test (TeLPI; Alves, Simões, \& Martins, 2009), the Subjective Memory Complaints scale (SMC; Schmand, Jonker, Hooijer, \& Lindeboom, 1996), and the Geriatric Depression Scale (GDS-30; Yesavage et al., 1983). The MoCA was never used for diagnostic purposes.

The control group was composed of cognitively healthy members of the community, living in Portugal. This group was selected from the database of the MoCA's normative study for the Portuguese population to match each patient on variables that were found to be predictive of the MoCA's performance (educational level and age; Freitas, Simões, Alves, \& Santana, 2011), and additionally on gender. Details of the controls' recruitment procedure, inclusion and exclusion criteria, and neuropsychological assessment have been described in a previous study (Freitas et al., 2011).

An informed consent was obtained from all the participants after the research aims, procedures, and confidentiality requirements were fully disclosed by a member of the research team. For the patients who were not capable of providing the informed consent, a legal representative 
fulfilled that requirement on their behalf. The present research complied with the ethical guidelines for human experimentation stated in the Declaration of Helsinki and was approved by the Ethics Board of Coimbra University Hospital, by the "Fundação para a Ciência e Tecnologia" [Portuguese Foundation for Science and Technology] and by the Faculty of Psychology and Educational Sciences Scientific Committee.

\section{Material and Neuropsychological Testing}

Each participant was assessed in a single session by a neuropsychologist experienced in evaluating patients with dementia. In the clinical interview, the demographic and clinical data were collected through a complete sociodemographic questionnaire, an inventory of past habits and of the current clinical health status as well as of the medical history. Following this, the same neuropsychologist administered the MMSE (Folstein et al., 1975; Guerreiro, 1998) and the MoCA (Nasreddine et al., 2005; Simões et al., 2008), in that fixed order, to all subjects. Both the MMSE and the MoCA are in paper-and-pencil format and are scored out of a possible total of 30 points, with higher scores indicating better cognitive performance. The MoCA was developed to screen milder forms of cognitive impairment through the assessment of six cognitive domains: (1) executive functions; (2) visuospatial abilities; (3) short-term memory; (4) language; (5) attention, concentration, and working memory; and (6) temporal and spatial orientation (Nasreddine et al., 2005). In the current study, the MoCA's total score refers to the raw score without any correction points for education effects. Nasreddine and collaborators (2005) suggest a correction point for individuals with 12 or less years of education. However, the Portuguese population has a considerable lower education level in comparison with the original study population. Due to this major difference, the correction point is not entirely applicable to the Portuguese population. The norms for the Portuguese population were calculated and stratified according to different educational levels (Freitas et al., 2011).

\section{Statistical Analysis}

Statistical analyses of the data were carried out using the Statistical Package for the Social Sciences (SPSS, version 19.0) (IBM SPSS, Chicago, IL). Descriptive statistics were used for the sample's characterization. The $\chi^{2}$ test, the two-sample $t$ test, the one-way between-groups analysis of variance (ANOVA), and the Tukey HSD and Bonferroni post hoc test were conducted to explore group differences. The Cronbach's alpha was considered as an index of internal consistency. The Pearson correlation coefficients were used for analyses of convergent validity, and to explore the correlations between items, cognitive domains and total scores.

The diagnostic accuracy of the MoCA and the MMSE for the prediction of the clinical diagnosis of $\mathrm{VaD}$ was assessed through the receiver operating characteristics (ROC) curve analysis, implemented in MedCalc (version 11.6) (MedCalc Software, Mariakerke), with larger areas under the curve (AUC) indicating better diagnostic accuracy. The ROC curves were compared according to the AUC comparison method of Hanley and McNeil (1983). The optimal cutoff points for each screening instrument that yielded the highest Youden index were selected, with higher Youden indices indicating maximum sensitivity and specificity. To analyze the predictive value of the tests, for each cutoff point, we calculated the sensitivity (the probability of subjects with cognitive impairment having a positive result), specificity (the probability of subjects without cognitive impairment having a negative test), positive predictive value (PPV, the probability of disease in subjects with a positive result), negative predictive value (NPV, probability of a "lack of disease" classification in subjects with a negative result), and classification accuracy (probability of correct classification of subjects, with or without cognitive impairment).

\section{RESULTS}

\section{Sample Characterization}

The characteristics of the study sample, and in more detail of all subgroups, are presented in Table 1. For this description, were considered: sample size, gender, age, educational level, MMSE score, and MoCA score.

As mentioned above, the participants in the three groups were matched based on gender, age, and educational level. Additionally, the $\mathrm{VaD}$ and $\mathrm{AD}$ patients were also matched based on their MMSE scores. In fact, as seen on Table 1, there are no gender differences between the three groups. Likewise, no statistically significant differences were found based on age $(F(2,99)=.095 ; p=.910)$ or educational level $(F(2,99)=.021 ; p=.979)$. Furthermore, statistically significant differences in MMSE scores were not observed between clinical groups $(t(66)=.826 ; p=.412)$, which suggests similarities in terms of cognitive decline severity.

\section{Psychometric Properties}

Cronbach's alpha of the MoCA as an index of internal consistency was 0.908 for the total study sample, confirming the overall reliability of the scale when used to examine Portuguese subjects. Regarding the analysis of MoCA items that could be eliminated to increase consistency, the results indicated that none should be excluded. This reliability coefficient was also computed for each clinical group: $\alpha(\mathrm{VaD})=.825$ and $\alpha(\mathrm{AD})=.806$. The respective values for the MMSE were: $\alpha($ total sample $)=.807, \alpha(\mathrm{VaD})=.771$, and $\alpha(\mathrm{AD})=.804$.

The correlations between the MoCA scores and the MMSE scores were significant and positive in the total sample $(r=.741 ; p<.001)$ and in the $\mathrm{VaD}$ group $(r=.782 ; p<.001)$, which is indicative of convergent validity. Regarding to the MoCA cognitive domains, we found significant and positive 
Table 2. Correlations of the scores on cognitive domains and the MoCA total score

\begin{tabular}{lclc}
\hline \hline & $\mathrm{VaD}$ & $\mathrm{AD}$ & Control \\
\hline Executive Functions & $.669^{* *}$ & $.453^{* *}$ & $.679^{* *}$ \\
Visuospatial Abilities & $.619^{* *}$ & $.428^{*}$ & $.664^{* *}$ \\
Memory & $.495^{* *}$ & $.458^{* *}$ & $.287^{*}$ \\
Language & $.754^{* *}$ & $.741^{* *}$ & $.562^{* *}$ \\
Attention, Concentration, & $.780^{* *}$ & $.813^{* *}$ & $.688^{* *}$ \\
$\quad$ and Working Memory & & & \\
Orientation & $.556^{* *}$ & $.698^{* *}$ & $.463^{* *}$ \\
\hline \hline
\end{tabular}

Note. $\mathrm{VaD}=$ group of patients with Vascular Dementia; $\mathrm{AD}=$ group of patients with Alzheimer's disease; Control = group of cognitively healthy adults; MoCA $=$ Montreal Cognitive Assessment.

$* * p<.01$

$* p<.05$.

correlations between each cognitive domain score and the total score of the scale for all three groups (Table 2), which is suggestive of construct-related validity. Additionally, regarding the correlation coefficients of each item within the cognitive domains, overall items showed a significant and positive correlation with the respective domain. The exceptions are the camel, second subtraction, place, and city items, which showed no significant correlation with any domain due to the lower variance of the results (hit rates: camel $=79.4$; second subtraction $=23.5$; place $=97.1$; city $=100$ ). Furthermore, the cube's copy showed a significant correlation with the visuospatial domain $(r=.452 ; p<.001)$ and with the executive function domain $(r=.491 ; p<.001)$.

\section{Group Differences}

Comparing the MoCA total scores of the three groups, statistically significant differences can be observed $(F(2,99)=78.121$; $p<.001)$. However, according to post hoc tests, these differences occurred between the control group and both clinical groups, with higher performance seen in the healthy participants. No differences were found between $\mathrm{VaD}$ and $\mathrm{AD}$ groups, which were previously matched based on their MMSE scores. As expected, a more detailed analysis regarding subscores of the MoCA cognitive domains revealed that the healthy participants had higher performances in all cognitive domains than both clinical groups. The scores on the cognitive domains for all three groups are presented on Table 3. There were no statistically significant differences between the $\mathrm{VaD}$ and $\mathrm{AD}$ patients at the level of the cognitive domains of the MoCA, nor at the level of some tasks' sub-scores, such as clock drawing, naming tasks, subtraction, and abstraction tasks. Additionally, no differences in the number of the words stated in the phonemic fluency task were found.

Considering the abbreviated version of the MoCA proposed by the NINDS-CSN VCI Harmonization Standards criteria (Hachinski et al., 2006) as a 5-min protocol (total score $=12$ ), no statistically significant differences were found between $\mathrm{VaD}(5.50 \pm 1.93)$ and $\mathrm{AD}$ patients $(4.65 \pm 1.89)$. Nevertheless, the short version of the MoCA discriminates control subjects $(9.32 \pm 1.408)$ from both clinical groups, according to the post hoc tests $(F(2,99)=68.295 ; p<.001)$.

\section{Cutoff Points}

The receiver operating characteristics (ROC) curve analysis and the predictive values were computed to evaluate the diagnostic accuracy of MoCA to discriminate $\mathrm{VaD}$ patients from cognitively healthy adults. First, the diagnostic accuracy of the MoCA full version was compared with the diagnostic accuracy of the MMSE; second, the MoCA short version was compared with the MMSE; and then, the MoCA full version was compared with the short version (Hachinski et al., 2006).

The MoCA full version discriminant potential for $\mathrm{VaD}$ was excellent, with an AUC of .950 (95\% confidence interval $=.868-.988$ ), while the MMSE demonstrated an AUC of .860 (95\% confidence interval $=.754-.932)$. These AUCs are significantly different $(Z=2.142 ; p=.0322)$, according to the AUC comparison method of Hanley and McNeil (1983), indicating different classificatory accuracy of the instruments. Graphic representations of the ROC curves are provided in Figure 1. Similarly, the MoCA short version also reveals an excellent discriminant potential for $\mathrm{VaD}$ with an AUC of .936 (95\% confidence interval $=.879-.992)$, although not significantly different from the MMSE results ( $Z=1.774 ; p=.076$ ), according to the AUC comparison method of Hanley and McNeil (1983). The respective graphic representations of the ROC curves are provided in Figure 2.

Table 3. Scores on the cognitive domains

\begin{tabular}{lccr}
\hline \hline & VaD & AD & Control \\
\hline Executive Functions & $0.59 \pm 0.988$ & $0.59 \pm 0.657$ & $2.15 \pm 1.019$ \\
Visuospatial Abilities & $1.41 \pm 0.892$ & $1.24 \pm 0.606$ & $3.09 \pm 1.055$ \\
Memory & $0.59 \pm 1.019$ & $0.29 \pm 0.676$ & $3.09 \pm 1.379$ \\
Language & $2.85 \pm 1.282$ & $2.38 \pm 1.457$ & $4.24 \pm 1.281$ \\
Attention, Concentration, and Working Memory & $2.85 \pm 1.760$ & $2.79 \pm 1.647$ & $4.85 \pm 1.209$ \\
Orientation & $4.82 \pm 1.267$ & $4.24 \pm 1.437$ & $5.88 \pm 0.327$ \\
\hline \hline
\end{tabular}

Note. $\mathrm{VaD}=$ group of patients with Vascular dementia; $\mathrm{AD}=$ group of patients with Alzheimer's disease; Control = group of cognitively healthy adults.

Data are presented as mean \pm standard deviation. 


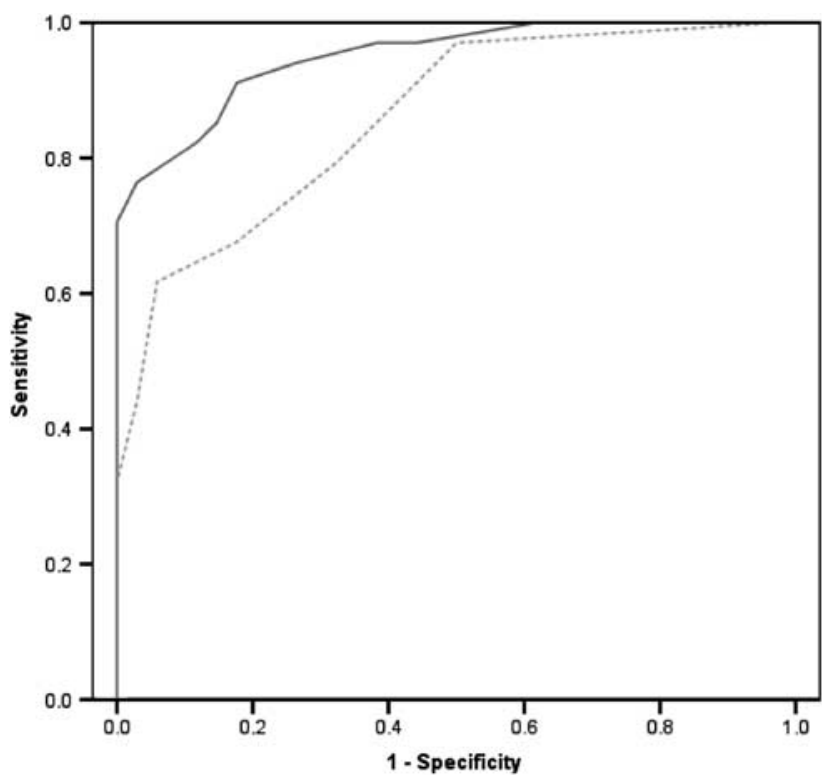

Fig. 1. Receiver operating characteristics (ROC) curve analysis of the MoCA full version (continuous line) and MMSE (dashed line) to detect $\mathrm{VaD}$ patients. MoCA $=$ Montreal Cognitive Assessment; MMSE $=$ Mini Mental State Examination; $\mathrm{VaD}=$ Vascular Dementia .

Nevertheless, in both comparisons it can be observed that the MoCA's ROC curves (full and short versions) fully include the curve for the MMSE; this is a clear indication that there is always a cutoff for the MoCA, with higher sensitivity and specificity, for any cutoff chosen for the MMSE. Comparing the AUCs of the MoCA full and short versions $(\mathrm{AUC}=.936 ; 95 \%$ confidence interval $=.849-.981)$, no statistically significant differences were found $(Z=.488$;

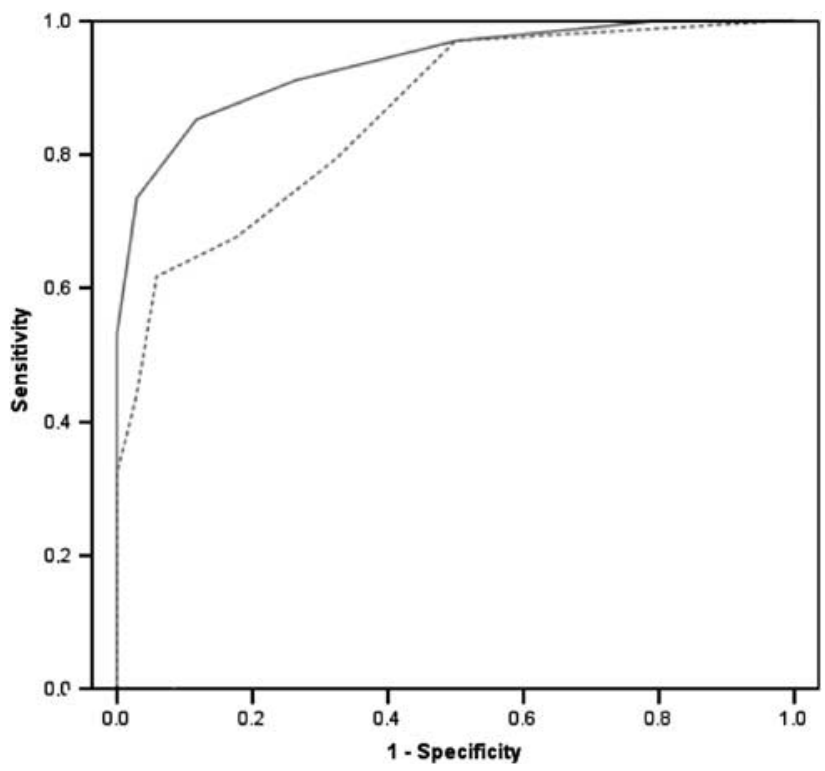

Fig. 2. Receiver operating characteristics (ROC) curve analysis of the MoCA short version (continuous line) and MMSE (dashed line) to detect $\mathrm{VaD}$ patients. MoCA = Montreal Cognitive Assessment; MMSE $=$ Mini Mental State Examination; $\mathrm{VaD}=$ Vascular Dementia.

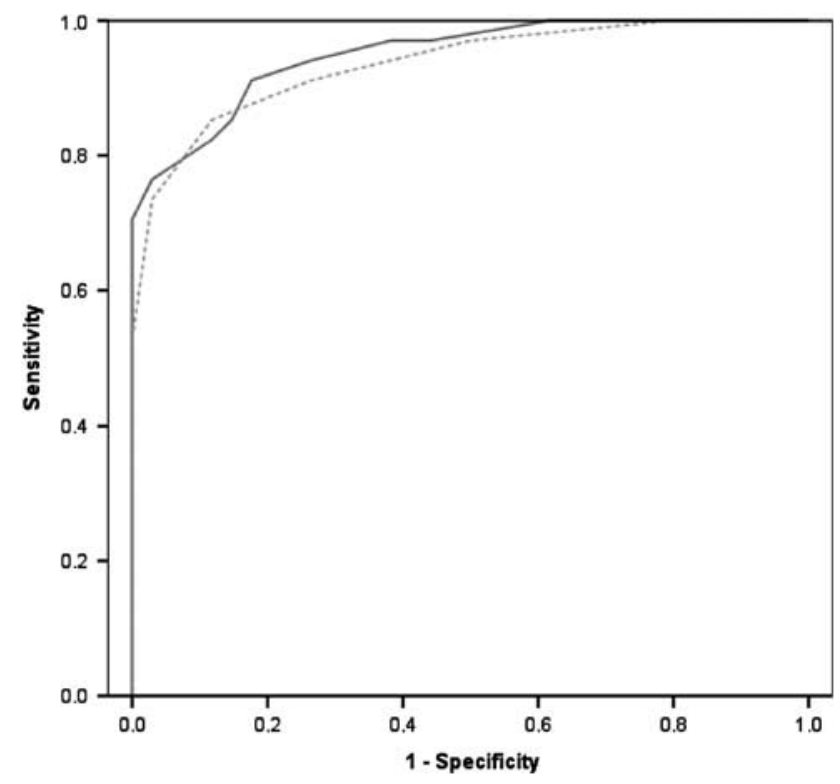

Fig. 3. Receiver operating characteristics (ROC) curve analysis of the MoCA full version (continuous line) and MoCA short version (dashed line) to detect $\mathrm{VaD}$ patients. $\mathrm{MoCA}=$ Montreal Cognitive Assessment; MMSE $=$ Mini Mental State Examination; $\mathrm{VaD}=$ Vascular Dementia

$p=.6256)$, according to the AUC comparison method of Hanley and McNeil (1983). Graphic representations of the respective ROC curves are provided in Figure 3. The optimal cutoff points for maximum accuracy (Youden index) and the respective values of sensitivity, specificity, PPV, NPV, and classification accuracy are described for both instruments in Table 4.

\section{DISCUSSION}

Accurate screening of the early stages of $\mathrm{VaD}$ assumes extreme importance, considering the high prevalence rates of vascular cognitive impairment and the available primary and secondary prevention strategies. The MMSE reveals limitations in effective cognitive assessment of patients with $\mathrm{VaD}$ because it does not include tasks for the evaluation of the main area of compromise: executive dysfunction. Thus, this study aims to validate the MoCA, as well as the short version of the MoCA proposed by NINDS-CSN VCI Harmonization Standards, as a 5-min protocol for cognitive screening of $\mathrm{VaD}$ patients.

The results of the present study demonstrate that the $\mathrm{MoCA}$ is a psychometrically valid and reliable instrument for the cognitive screening of $\mathrm{VaD}$ patients. The internal consistency was high, confirming the overall reliability of the scale when used to examine Portuguese patients. Furthermore, the significant and positive correlations between the MoCA scores and the MMSE scores are suggestive of convergent validity. On the other hand, the significant and positive correlations between each MoCA's cognitive domain and the MoCA total score are suggestive of construct-related validity. 
Table 4. Diagnostic classification accuracy

\begin{tabular}{lcccccc}
\hline \hline & Cutoff & Sensitivity & Specificity & PPV & NPV & Classification accuracy \\
\hline MoCA Total Scale & $<17$ & 77 & 97 & 96 & 81 & 87 \\
MoCA Short Version & $<8$ & 85 & 88 & 88 & 86 & 87 \\
MMSE & $<26$ & 62 & 94 & 91 & 71 & 78 \\
\hline
\end{tabular}

Note. Sensitivity, Specificity, PPV, NPV, and Classification Accuracy values were expressed in percentage. Cutoff values indicate the minimum score required for absence of signal.

MoCA $=$ Montreal Cognitive Assessment (maximum score $=30$ ); MoCA Short Version $=$ abbreviated version proposed by the NINDS-CSN VCI Harmonization Standards criteria (maximum score $=12$ ); MMSE = Mini Mental State Examination (maximum score $=30$ ); AUC $=$ area under the operating characteristic curve; $\mathrm{PPV}=$ positive predictive value; $\mathrm{NPV}=$ negative predictive value.

Finally, all items showed a significant correlation with their respective domains, with the exception of camel, second subtraction, place, and city items; these showed no significant correlation with any domain, possibly due to the lower variance of the items in this sample. Furthermore, we hypothesize that the significant correlation found between the cube's copy item and executive function domain, beyond its correlation with the visuospatial domain, is explained by the threedimensional nature of the drawing; it involves the use of planning, sequencing and attentional skills.

For the MoCA's discriminant capacity analysis, participants in the three groups ( $\mathrm{VaD}, \mathrm{AD}$, and controls) were matched for gender, age, and educational level; the two clinical groups were also matched based on MMSE scores to ensure comparable levels of severity of cognitive decline, which we consider be essential for a reliable comparative analysis, although it may have a minor effect on the correlation coefficient between the two tests. The results showed that both the full and short versions of the MoCA efficiently discriminate the cognitively healthy adults from both clinical groups. The performance of the healthy participants in all MoCA cognitive domains was also significantly higher than that of both clinical groups. However, no statistically significant differences were found between $\mathrm{VaD}$ and $\mathrm{AD}$ patients using both MoCA versions. Additionally, no differences were found between $\mathrm{VaD}$ and $\mathrm{AD}$ patients' performances in the different cognitive domains of the MoCA, or at the level of some tasks' sub-scores (clock drawing, naming tasks, subtraction, and abstraction tasks). These findings were convergent with the results of several prior studies, which have failed to identify significant differences in neuropsychological performance between $\mathrm{VaD}$ and $\mathrm{AD}$ patients (Braaten, Parsons, McCue, Sellers, \& Burns, 2006; Desmond, 2004). Given the characteristics of the MoCA (namely the more comprehensive assessment of major cognitive domains as executive function, short-term memory, languages abilities, and visuospatial processing) and the findings of previous studies from our group, we hypothesized that it could capture any difference in the cognitive profile of these clinical groups. In the three validation studies developed by our research group (Freitas, Simões, Marôco, Alves, \& Santana, 2012; Freitas, Simões, Alves, Duro, \& Santana, manuscript submitted for publication; Freitas, Simões, Alves, \& Santana, 2012), the MoCA's sensitivity and discriminant capacity at level of cognitive domains were well-demonstrated. Thus, we believe that the undifferentiated performance founded in this study may reflect the very close cognitive profile between $\mathrm{VaD}$ and the $\mathrm{AD}$ patients more so than the instrument's inability to capture cognitive profiles.

The MoCA scores displayed excellent diagnostic accuracy in the discrimination of $\mathrm{VaD}$ symptoms from age-related changes in cognitively healthy elderly adults. The MoCA full version scores exhibits an AUC significantly higher than that of the MMSE's AUC; this confirms the comparative superior classificatory accuracy of the MoCA full version. The optimal cutoff point on the MoCA for $\mathrm{VaD}$ patients, allowing maximum sensitivity and specificity, was below 17 points. This cutoff point is equivalent to the optimal cutoff for $\mathrm{AD}$, as established by our previous validation study (Freitas, et al., 2012), as well as for Frontotemporal Dementia-behavioralvariant patients (Freitas et al., submitted). With this cutoff point, the MoCA showed good sensitivity (77\%), excellent specificity (97\%) and PPV (96\%), and good NPV (81\%) and classification accuracy (87\%). With an optimal cutoff point of below 26 points, the MMSE had consistently lower respective values, namely in sensitivity (62\%) and classificatory accuracy (78\%); this suggests that the MMSE is not a good option as cognitive screening instrument for $\mathrm{VaD}$, as some authors have claimed (Hachinski et al., 2006; Moorhouse \& Rockwood, 2008; Pendlebury et al., 2010; Román, 2003). The main reasons for the MoCA's higher results at this level were the inclusion of the executive function assessment and the consideration of more complex tasks to measure short-term memory, language, attention, concentration, working memory, and visuospatial skills.

The MoCA's short version, proposed by NINDS-CSN VCI Harmonization Standards (Hachinski et al., 2006) as a 5-min protocol, also demonstrated excellent diagnostic accuracy in discriminating $\mathrm{VaD}$ patients from the control participants. The AUCs of the MoCA short and full versions scores revealed no statistically significant differences, which indicate that the MoCA short version is a valid abbreviated option, useful in clinical settings with time constraints. However, contrary to what occurred with the MoCA full version, no significant differences were found in comparison with the MMSE, although the MoCA short version's ROC curve fully include the curve for the MMSE, which indicate that there is always a cutoff for the MoCA short version, with 
higher sensitivity and specificity, for any cutoff chosen for the MMSE. With an optimal cutoff point of below 8 points, the MoCA short version showed high sensitivity $(85 \%)$, specificity (88\%), PPV (88\%), NPV (86\%), and classification accuracy $(87 \%)$.

Due to the clinical heterogeneity of the VCI conditions, this investigation was carried out in a homogeneous sample of $\mathrm{VaD}$ patients. Patients who did not meet the criteria for dementia or with mixed dementia classification were excluded. The rigorous procedures adopted by the multidisciplinary team ensure the correct diagnosis of patients according to the international criteria for $\mathrm{VaD}$ (Román et al., 1993). Major methodological strengths are the comprehensive clinical evaluation with routine laboratory exams, strict and rigorous imaging inclusion criteria, a comprehensive neuropsychological assessment battery, and several exclusion criteria for patients' selection at the outset. Although some studies conducted in cerebrovascular and cardiovascular patients support the validity, reliability and sensitivity of the MoCA, there are few previous studies using a homogeneous group of $\mathrm{VaD}$ patients to confirm those results and no studies of validation of the MoCA short version.

The main strengths of this study are: (1) the homogeneity of the $\mathrm{VaD}$ group; (2) the homogeneity of the samples in terms of group size, gender, age, educational level, and severity of cognitive decline in the clinical groups, which allowed a more clear analysis and minimized the influence of individual and methodological variables; (3) the abovementioned rigorous procedures adopted to ensure a probable diagnosis of $\mathrm{VaD}$ and $\mathrm{AD}$; (4) the well-characterized control group, composed of cognitively healthy adult members of the community; and (5) the reduction of inter-rater variability because all participants were assessed by one of two expert neuropsychologists.

Some limitations of this study must be addressed, however. First, as we intended to include and obtain results from $\mathrm{VaD}$ patients, the generalization of these results to other VCI subtypes should be performed with caution. Another point to mention is that the scores of the MoCA short version were computed after the complete administration of the MoCA, rather than resulting from a second separate administration. Despite there are no guidelines for the administration order of short version items, in fact we did not perform a separate administration of both versions. So although we should to admit this procedure as a limitation, we believe that the impact is limited because all additional items are very brief tasks, with short and immediate answers and the full MoCA is itself a short test. Another issue that needs to be pointed out is the use of the MMSE as both a cutoff for severity level's restriction and a variable of interest, making the results incorporating the MMSE somewhat circular in nature. However, unfortunately it was not possible to consider a third instrument for restricting the severity level on the study due to constraints in the department's functioning and neuropsychological assessment protocol currently used. The severity level's restriction was adopted because the MoCA was designed as a test for mild to moderate cognitive decline. The complexity of the tasks that compose the test is not adequate for patients with a more pronounced cognitive decline. Finally, because this is the first study that (i) validated the MoCA short version for $\mathrm{VaD}$ patients, (ii) compared a shortened form to the original form of the MoCA, and (iii) computed the cutoff points for both MoCA short and full versions for the screening of $\mathrm{VaD}$ patients, a comparative analysis of this results with other studies cannot be performed.

Some future considerations: the need for (i) more studies conducted in different cultural contexts other than the Portuguese, (ii) studies with separate administrations of the two versions of the MoCA; and (iii) studies with larger sample sizes, which allow that the each clinical group is divided into two subgroups, high and low scorers on the MMSE, to further confirm the present findings.

In conclusion, the MoCA full and short versions proved to be valid, reliable, sensitive and accurate measures of cognitive impairment in $\mathrm{VaD}$. Compared with the widely used MMSE, this study produced evidence of the overall superiority of both MoCA versions in terms of diagnostic accuracy, confirming its great potential and usefulness for the brief cognitive assessment of $\mathrm{VaD}$ patients.

\section{ACKNOWLEDGMENTS}

This work was supported by the Fundação para a Ciência $e$ Tecnologia [Portuguese Foundation for Science and Technology] through of a PhD fellowship (SFRH/BD/38019/2007) and PIC/IC/ $83206 / 2007$. All authors of this study declare that there are no conflicts of interest.

\section{REFERENCES}

Aggarwal, A., \& Kean, E. (2010). Comparison of the Folstein Mini Mental State Examination (MMSE) to the Montreal Cognitive Assessment (MoCA) as a cognitive screening tool in an inpatient rehabilitation setting. Neuroscience \& Medicine, 1, 39-42.

Alves, L., Simões, M. R., \& Martins, C. (2009). Teste de Leitura de Palavras Irregulares (TeLPI) [Reading Word Irregular Test]. Coimbra, Portugal: Serviço de Avaliação Psicológica da Faculdade de Psicologia e de Ciências da Educação da Universidade de Coimbra [Psychological Assessment Department, Faculty of Psychology and Educational Sciences, University of Coimbra].

American Psychiatric Association. (2000). Diagnostic and Statistical Manual of Mental Disorders (4th ed. - Text Revised). Washington, DC: American Psychiatric Association Press.

Braaten, A.J., Parsons, T.D., McCue, R., Sellers, A., \& Burns, W.J. (2006). Neurocognitive differential diagnosis of dementing diseases: Alzheimer's disease, vascular dementia, frontotemporal dementia, and major depressive disorder. International Journal of Neuroscience, 116, 1271-1293.

Canadian Study of Health and Aging Working Group. (1994). Canadian study of health and aging: Study methods and prevalence of dementia. Canadian Medical Association Journal, 150(6), 899-913.

Cumming, T.B., Bernhardt, J., \& Linden, T. (2011). The Montreal Cognitive Assessment: Short cognitive evaluation in a large stroke trial. Stroke, 42, 2642-2644. doi:10.1161/ STROKEAHA.111.619486 
Desmond, D.W. (2004). Vascular dementia. Clinical Neuroscience Research, 3, 437-448.

Dong, Y., Sharma, V.K., Chan, B.P., Venketasubramanian, N., Teoh, H.L., Seet, R.C., ... Chen, C. (2010). The Montreal Cognitive Assessment (MoCA) is superior to the Mini-Mental State Examination (MMSE) for the detection of vascular cognitive impairment after acute stroke. Journal of the Neurological Sciences, 299(1-2), 15-18.

Folstein, M., Folstein, S., \& McHugh, P. (1975). Mini-Mental State: A practical method for grading the cognitive state of patients for the clinician. Journal of Psychiatric Research, 12(3), 189-198.

Freitas, S., Santana, I., \& Simões, M.R. (2010). The sensitivity of the MoCA and MMSE to cognitive decline: A longitudinal study. Alzheimer's \& Dementia, 6(4), S353-S354.

Freitas, S., Simões, M.R., Alves, L., Duro, D., \& Santana, I. (2012). Montreal Cognitive Assessment (MoCA): Validation study for Frontotemporal Dementia. Journal of Geriatric Psychiatry and Neurology, in press.

Freitas, S., Simões, M.R., Alves, L., \& Santana, I. (2011). Montreal Cognitive Assessment (MoCA): Normative study for the Portuguese population. Journal of Clinical and Experimental Neuropsychology, 33(9), 989-996.

Freitas, S., Simões, M. R., Alves, L., \& Santana, I. (2012). Montreal Cognitive Assessment (MoCA): Validation study for Mild Cognitive Impairment and Alzheimer's disease. Alzheimer Disease and Associated Disorders, doi:10.1097/WAD.0b013e3182420bfe

Freitas, S., Simões, M.R., Marôco, J., Alves, L., \& Santana, I. (2012). Construct validity of the Montreal Cognitive Assessment (MoCA). Journal of International Neuropsychology Society, 18, 1-9.

Garrett, K.D., Paul, R.H., Libon, D.J., \& Cohen, R.A. (2004). Defining the diagnosis of vascular dementia. Applied Neuropsychology, 11(4), 202-207.

Gauthier, S., Patterson, C., Gordon, M., Soucy, J., Schubert, F., \& Leuzy, A. (2011). Commentary on "Recommendations from the National Institute on Aging-Alzheimer's Association workgroups on diagnostic guidelines for Alzheimer's disease. ": A Canadian perspective. Alzheimer's \& Dementia, , 7, 330-332.

Guerreiro, M. (1998). Contributo da Neuropsicologia para o estudo das demencias [Contribution of Neuropsychology to the study of dementia] (Unpublished doctoral dissertation). University of Lisbon, Lisbon.

Hachinski, V., \& Bowler, J.V. (1993). Vascular dementia. Neurology, 43, 2159-2160.

Hachinski, V., Iadecola, C., Petersen, R.C., Breteler, M.M., Nyenhuis, D.L., Black, S.E., ... Leblanc, G.G. (2006). National Institute of Neurological Disorders and Stroke - Canadian Stroke Network Vascular Cognitive Impairment Harmonization Standards. Stroke, 37, 2220-2241.

Hachinski, V., Iliff, L.D., Zihlka, E., Du Boulay, G.H., McAllister, V.L., Marshall, J., ... Symon, L. (1975). Cerebral blood flow in dementia. Archives of Neurology, 32, 632-637.

Hachinski, V., Lassen, J., \& Marshall, J. (1974). Multi-infarct dementia: A cause of mental deterioration in the elderly. Lancet, 2, 207-210.

Hanley, J.A., \& McNeil, B.J. (1983). A method of comparing the areas under receiver operating characteristic curves. Radiology, $148,839-843$.

Hughes, C.P., Berg, L., Danziger, W.L., Coben, L.A., \& Martin, R.L. (1982). A new clinical scale for the staging of dementia. The British Journal of Psychiatry, 140, 566-572.

Ihara, M., Okamoto, Y., \& Takahashi, R. (2012). Suitability of the Montreal Cognitive Assessment versus the Mini-Mental State
Examination in detecting Vascular Cognitive Impairment. Journal of Stroke and Cerebrovascular Diseases. doi:10.1016/ j.jstrocecerebrovasdis.2012.01.001 [Epub ahead of print].

Ihl, R., Frölich, T.D., Martin, E.M., \& Maurer, K. (1992). Differential validity of psychometric tests in dementia of Alzheimer type. Psychiatry Research, 44, 93-106.

Ikeda, M., Hokoishi, K., Maki, N., Nebu, A., Tachibana, N., Komori, K., ... Tanable, H. (2001). Increased prevalence of vascular dementia in Japan: A community-based epidemiological study. Neurology, 57, 839-844.

Ismail, Z., Rajji, T.K., \& Shulman, K.I. (2009). Brief cognitive screening instruments: An update. International Journal of Geriatric Psychiatry, 25(2), 111-120. doi:10.1002/gps.2306

Jacova, C., Kertesz, A., Blair, M., Fisk, J.D., \& Feldman, H.H. (2007). Neuropsychological testing and assessment for dementia. Alzheimer's Dementia, 3(4), 299-317. doi:10.1016/ j.jalz.2007.07.011

Jorm, A., \& O'Brien, J. (2004). The epidemiology of vascular dementia: An overview and commentary. In J. O'Brien, D. Ames, L. Gustafson, M. Folstein, \& E. Chiu (Eds.), Cerebrovascular disease, cognitive impairment and dementia (2nd ed., pp. 95-100). London: Martin Dunitz.

Lamar, M., Price, C.C., Giovannetti, T., Swenson, R., \& Libon, D.J. (2010). The dysexecutive syndrome associated with ischaemic vascular disease and related subcortical neuropathology: A Boston process approach. Behavioural Neurology, 22, 53-62. doi:10.3233/BEN-2009-0237

Lobo, A., Launer, L.J., Fratiglioni, L., Andersen, K., Di Carlo, A., Breteler, M.M., ... Hofman, A. (2000). Prevalence of dementia and major subtypes in Europe: A collaborative study of population-based cohorts. Neurology, 54(Suppl. 5), S4-S9.

Lobo, A., Martinez-Lage, J., Soininen, H., \& Hofman, A. (2000). Incidence of dementia and major subtypes in Europe: A collaborative study of population-based cohorts. Neurology, 54(Suppl. 5), S10-S15.

Lonie, J.A., Tierney, K.M., \& Ebmeier, K.P. (2009). Screening for Mild Cognitive Impairment: A systematic review. International Journal of Geriatric Psychiatry, 24(9), 902-915. doi:10.1002/gps.2208

Looi, J.C., \& Sachdev, P.S. (1999). Differentiation of vascular dementia from AD on neuropsychological tests. Neurology, 53, 670-678.

Martinić-Popović, I., Šerić, V., \& Demarin, V. (2006). Early detection of mild cognitive impairment in patients with cerebrovascular disease. Acta Clinica Croatica, 45, 77-85.

McKhann, G., Drachman, D., Folstein, M., Katzman, R., Price, D., \& Stadlan, E.M. (1984). Clinical diagnosis of Alzheimer's disease: Report of the NINCDS-ADRDA Work Group under the auspices of the Department of Health and Human Services Task Force on Alzheimer's disease. Neurology, 34, 939-944.

Moorhouse, P., \& Rockwood, K. (2008). Vascular cognitive impairment: Current concepts and clinical developments. Lancet Neurology, 7, 246-255.

Mungas, D., Reed, B.R., \& Kramer, J.H. (2003). Psychometrically matched measures of global cognition, memory, and executive function for assessment of cognitive decline in older persons. Neuropsychology, 17(3), 380-392.

Nasreddine, Z., Phillips, N.A., Bédirian, V., Charbonneau, S., Whitehead, V., Collin, I., ... Chertkow, H. (2005). The Montreal Cognitive Assessment, MoCA: A brief screening tool for Mild Cognitive Impairment. Journal of American Geriatrics Society, 53(4), 695-699.

O'Brien, J., Reisberg, B., \& Erkinjuntti, T. (2003). Vascular burden of the brain. International Psychogeriatrics, 15(1), 7-10. 
Pendlebury, S.T., Cuthbertson, F.C., Welch, S.J., Mehta, Z., \& Rothwell, P.M. (2010). Underestimation of cognitive impairment by Mini-Mental State Examination versus the Montreal Cognitive Assessment in patients with transient ischemic attack and stroke: A population-based study. Stroke, 41, 1290-1293.

Pirscoveanu, D., Tudorica, V., Matcau, L., Zaharia, C., Matcau, D., \& Ene, L. (2009). Six months follow-up study on cognitive performances in patients with ischemic vascular events. Timisoara Medical, 59(1), 81-85.

Reed, B.R., Mungas, D.M., Kramer, J.H., Betz, B.P., Ellis, W., Vinters, H.V., ... Chui, H.C. (2004). Clinical and neuropsychological features in autopsy-defined vascular dementia. The Clinical Neuropsychologist, 18(1), 63-74.

Román, G.C. (2003). Vascular dementia: Distinguishing characteristics, treatment, and prevention. Journal of American Geriatrics Society, 51, S296-S304.

Román, G.C., \& Royall, D.R. (1999). Executive control function: A rational basis for the diagnosis of vascular dementia. Alzheimer's Disease \& Associated Disorders, 13(Suppl. 3), S69-S80.

Román, G.C., Tatemichi, T.K., Erkinjuntti, T., Cummings, J.L., Masdeu, J.C., Garcia, J.H., ... Scheinberg, P. (1993). Vascular dementia: Diagnostic criteria for research studies-Report of the NINDS-AIREN International Workshop. Neurology, 43, 250-260.

Rosen, W.G., Mohs, R.C., \& Davis, K.L. (1984). A new rating scale for Alzheimer's Disease. American Journal of Psychiatry, 141, 1356-1364.

Santana, I. (2006). Demência Vascular [Vascular dementia]. In H. Firmino (Ed.), Psicogeriatria [Psychogeriatry] (pp. 413-426). Lisbon: Psiquiatria Clínica.

Schmand, B., Jonker, C., Hooijer, C., \& Lindeboom, J. (1996). Subjective memory complaints may announce dementia. Neurology, 46(1), 121-125.

Simões, M. R., Freitas, S., Santana, I., Firmino, H., Martins, C., Nasreddine, Z., \& Vilar, M. (2008). Montreal Cognitive
Assessment (MoCA): Versão portuguesa [Montreal Cognitive Assessment (MoCA): Portuguese version]. Coimbra, Portugal: Serviço de Avaliação Psicológica da Faculdade de Psicologia e de Ciências da Educação da Universidade de Coimbra [Psychological Assessment Department, Faculty of Psychology and Educational Sciences, University of Coimbra].

Tombaugh, T.N., \& McIntyre, N.J. (1992). The Mini-Mental State Examination: A comprehensive review. Journal of American Geriatrics Society, 40, 922-935.

Ueda, K., Kawano, H., Hasuo, Y., \& Fujishima, M. (1992). Prevalence and etiology of dementia in a Japanese community. Stroke, 23, 789-803.

van Straaten, E.C., Scheltens, P., Knol, D.L., van Buchem, M.A., van Dijk, E.J., Hofman, P.A., ... Barkhof, F. (2003). Operational definitions for the NINDS-AIREN criteria for Vascular dementia. Stroke, 34, 1907-1912.

Wind, A.W., Schellevis, F.G., van Staveren, G., Scholten, R.P., Jonker, C., \& van Eijk, J.T. (1997). Limitations of the Mini-Mental State Examination in diagnosing dementia in general practice. International Journal of Geriatric Psychiatry, 12, 101-108.

Wong, A., Kwan, P., Chan, A., Lam, W., Nyenhuis, D., Nasreddine, Z., \& Wong, L. (2008). The validity, reliability and utility of the Cantonese Montreal Cognitive Assessment (MoCA) in Chinese patients with confluent white matter lesions. Hong Kong Medical Journal, 14(6), FP1.

Wong, A., Xiong, Y.Y., Kwan, P.W., Chan, A.Y., Lam, W.W., Wang, K., ... Mok, V.C. (2009). The validity, reliability and clinical utility of the Hong Kong Montreal Cognitive Assessment (HK-MoCA) in patients with cerebral small vessel disease. Dementia and Geriatric Cognitive Disorders, 28, 81-87.

Yesavage, J.A., Brink, T.L., Rose, T.L., Lum, O., Huang, V., Adey, M., \& Leirer, V.O. (1983). Development and validation of a geriatric depression screening scale: A preliminary report. Journal of Psychiatric Research, 17(1), 37-49. 